\title{
Therapeutische Wirksamkeit ist kein dehnbarer Begriff
}

\section{Rolf Adler}

Prof. em. Dr. med., ehem. Chefarzt Innere Medizin C.L. Lory-Haus, Inselspital Bern

Wirksamkeit ist neben Zweckmässigkeit und Wirtschaftlichkeit ein Leitmotiv schweizerischer Gesundheitspolitik. Übertrifft eine Therapiewirkung die des Plazebos signifikant, steht sie zu Therapie-Nebenwirkungen in einem vernünftigen, zweckmässigen Verhältnis, und genügt sie wissenschaftlich anerkannter Methodik, so steht ihrer Eingliederung in die normale Medizin nichts im Wege.

Die von alternativer/komplementärer Seite (AK) oft vorgebrachte Meinung, ihre Ergebnisse entzögen sich den Messmethoden der Normalmedizin, ist nicht stichhaltig, denn die interessierenden Endpunkte einer Therapie, also Beseitigung von Symptomen, von Krankheiten, Morbidität, Mortalität, und Förderung des Gesundheitsgefühls, sind dieselben. Lediglich die statistisch verwendeten Methoden unterscheiden sich in bestimmten Fällen.

\section{Messskalen in der Normalmedizin}

In der normalen Medizin werden als Messskalen Intervall- und Rationalskalen verwendet. Eine Intervallskala besitzt zwischen den einzelnen Intensitätsschritten regelmässige Abstände. Für die Rationalskala gilt dasselbe, nur hat sie zusätzlich einen Nullpunkt. In einer Medizin, die psychische und soziale Begebenheiten einschliesst, können Nominal- und Ordinalskalen verwendet werden. Die Nominalskala klassiert nach Begriffen. Die Ordinalskala unterscheidet Schritte zwischen den einzelnen Intensitätsstufen nach $\mathrm{Zu}$ - und Abnahme, wobei die Abstände ungleich sein können. Als Beispiel: Der Plan der Londoner Untergrundbahnstationen kann mit genauen Kilometerabständen zwischen den einzelnen Stationen gezeichnet werden. Dem entsprechen Intervall- und Rationalskalen. Nehmen wir eine Gummimatrize, die wir in verschiedenen Richtungen zerren, vermögen wir die Abstände zwischen den Stationen nicht mehr nach Kilometern zu messen, aber die Reihenfolge der Stationen auf den einzelnen Linien bleiben die gleichen: So liegt Wembley auf der Metropolitan Line North immer noch näher an Baker Street als Northwood, und die Zwischenstationen bleiben dieselben.
Mein Artikel versucht zu klären, warum eine erstaunlich grosse Zahl von Ärzten, die in unserem aufgeklärten Zeitalter im Gymnasium und Studium mit wissenschaftlichem Denken vertraut gemacht worden sind, AK-Therapien selber verwenden oder die Patienten Ärzten zuweisen, die solche Therapien anwenden, obwohl die meisten biologisch resp. biopsychosozial unplausibel und deren Wirkung nie eindeutig nachgewiesen worden sind.

Zur Klärung dieser Frage nehme ich Homöopathie, anthroposophische Medizin und Neuraltherapie unter die Lupe. Warum? In den neunziger Jahren wurde ich von der Medizinischen Fakultät mit zwei Kollegen als Kommission aufgefordert, die Argumente für die Einrichtung einer Professur für AK-Medizin zu erarbeiten. Wir erkundigten uns, welche AK-Gebiete am häufigsten angewendet werden. Diese drei Gebiete wurden genannt. Wir legten der Fakultät unsere Ansicht dar: Ein Mitglied jedes dieser Gebiete hat während eines Jahres Einsitz in die Fakultätssitzungen mit Stimme, und übergibt den Sitz jährlich dem nächsten Gebiet. Eine Professur soll nicht eingerichtet werden beim Stand der wissenschaftlich zweifelhaften Ergebnisse der genannten Gebiete.

\section{Zur Homöopathie}

Seit zweihundert Jahren stützt sie sich auf zwei Grundsätze. Auf «Similia similibus curentur» und auf die Potenzierung, bei der der wirksame Stoff verdünnt wird, bis kein einziges Molekül mehr in der Lösung vorhanden zu sein braucht, weil durch Schlagen der Flüssigkeit in einem Ledersack auf einen Ledersattel die jeweilige Potenzstufe die Stoffeigenschaft dem Wasser aufprägt, das Wasser also eine Erinnerung an den Stoff behält. Ein Beispiel zu Similia: Wer Zwiebeln schneidet, beginnt zu tränen. Wer Heuschnupfen hat, tränt auch. Zwiebel in hoher Potenzierung wird Tränen beheben [1].

In der modernen Chemie gibt es keine Anhaltspunkte für die Beziehung zwischen Wirksamkeit und abnehmender Konzentration, auch nicht für das Erinnerungsvermögen von Wasser. Finden wir im Unbewussten des 
Arztes, den diese biologische Unplausibilität nicht hindert, Erklärungen [2]? Seit Sigmund Freud kennen wir die Arbeitsweisen der Psyche mit ihren Abwehr- und Bewältigungsvorgängen. In der Kleinkinderzeit beherrschen sie das Denken. Wir nennen diese Denkweise Primärprozess-Denken. Es richtet sich nach den Wünschen des Individuums und nicht nach den Geboten der Realität. Im Laufe der individuellen Entwicklung übernehmen Logik und Realitätsanpassung die Führung im Denken. Dies nennen wir Sekundärprozess-Denken. Die Psyche des Erwachsenen behält die Fähigkeit zum primärprozesshaften Denken und wendet es an, wenn den Wünschen nicht stattgegeben werden kann. Zu den primärprozesshaften Arbeitsweisen zählen wir das Verleugnen, das Verdrängen, das Projizieren, das Introjizieren, das Rationalisieren, das Bagatellisieren, die Umkehr ins Gegenteil, das Identifizieren, das Ersetzen durch Ähnliches und die Allmacht von Gedanken, d.h. magisches Denken und Handeln. Solange diese Denkweisen die Realitätsanpassung nicht stören, dienen sie der Bewältigung unseres Lebens. Werden sie unangepasst stark, können dem Individuum Nachteile erwachsen. So wird ein Student, der seine mangelhafte Examensvorbereitung beim Nichtbestehen des Examens verleugnet, sein Scheitern der Ungerechtigkeit des Examinators zuschreibt, bei der Wiederholung der Prüfung wieder versagen. Der Student, der seine schlechte Vorbereitung zur Kenntnis nimmt, wird besser arbeiten und die Examenswiederholung bestehen.

Ein Beispiel zum Verwenden des Ähnlichkeitsprinzips: Meine Tochter ist Oboistin und tritt mit dem bekannten Oboisten Kurt Meier auf. Ich möchte wissen, ob dieser beim weltberühmten Oboisten ... sein Name entfällt mir, ausgebildet worden ist. Ich versetze mich ins primärprozesshafte Denken und assoziiere frei. Dabei fällt mir Frutiger ein, dann Hottingerplatz und dann Holliger, Heinz. Frutiger ist dreisilbig und endet mit ger. Hottinger(platz) ist auch dreisilbig und beginnt mit Ho, weist einen Doppelkonsonanten auf und endet mit ger. Diese Eigenheiten der Namen führen zum Namen Holliger. Bei der Homöopathie finden wir den Ähnlichkeitsmechanismus im Similia. Die Umkehr ins Gegenteil zeigt sich in Wirksamkeitstszu- statt -abnahme beim Verdünnen. Das magische Denken und die Allmacht von Gedanken beobachten wir im Schlage-Zeremoniell.

Eine Erfahrung mit einem Forscherteam aus der Homöopathie soll dieses Denken illustrieren [3]. Sie haben eine randomisierte, doppelblinde Untersuchung an Konzentrations- und Aufmerksamkeitsgestörten Kindern durchgeführt. In einem offenen Vorversuch schliessen sie ein Drittel der Kinder, die auf das Verum nicht positiv reagieren, vom Hauptversuch aus. Die Gruppe A erhält in der ersten Phase das Verum, in der zweiten das Plazebo. Bei der Gruppe B ist es umgekehrt. Die leicht negative Wirkung des Verums in der ersten Phase bei der Hälfte der Versuchspersonen (Gruppe A) führen sie darauf zurück, dass sich die Eltern vorgestellt hätten, die Kinder erhielten in der ersten Phase das Plazebo (ein Interpretationsvorurteil). Die leicht positive Wirkung bei der andern Hälfte (B) in der zweiten (Verum-)Phase interpretieren sie als Beweis für die Wirkung der Homöopathie, obwohl der Effekt nur einen Punkt auf der Skala von 0 bis 10 beträgt, während er in der Vorphase um 8 war, was eindeutig für eine Plazebowirkung spricht (wieder ein Wunschdenken der Autoren). Ich möchte diese Fehler mit ihnen besprechen. Keine Antwort. Wir schreiben

In der modernen Chemie gibt es keine Anhaltspunkte für die Beziehung zwischen Wirksamkeit und abnehmender Konzentration.

dem Europ J Pediatrics unsere Kommentare [4], die akzeptiert und veröffentlicht werden. Ein Anruf beim Leiter der komplementärmedizinischen Abteilung eines Spitals und Mitglied der Forschergruppe wird nicht beantwortet. Er bezieht sich in einem schriftlichen Beitrag später an ein medizinisches Journal auf seine beispielhafte Studie. Wir konstatieren die Verleugnung der Realität und das Fortbestehen des Wunschdenkens, das jetzt Patienten-schädlich wird.

\section{Anthroposophische Medizin}

Hier lasse ich Ernst [1] sprechen, den besten Erforscher von AK-Therapien: «... is based on imagination, inspiration, intuition ... influenced by mystical, alchemistic, homoeopathic concepts ... it assumes metaphysical relations between planets, metals and human organs (magisches, projektives Denken) ... diseases are related to action (des Patienten) in previous lives; in order to redeem oneself ... best to live through them without conventional therapy ... employed are herbal extracts, art therapy, massage, exercise ... best known remedy is a fermented mistletoe extract ... it treats cancer ... is a parasitic plant ... kills the host ... striking resemblance to a malignant tumor, which also lives on his host and finally kills him/her ... therefore mistletoe can be used in cancer therapy (Ähnlichkeitsprinzip) ... biologically implausible ... efficacy remains unproven ... other elements of the anthroposophical concept not well researched ... mistletoe injections have been associated with a range of adverse effects ... most important risk is that of discarding conventional treatments ... anthroposophic medicine is 
biologically implausible, has not shown to be effective.» Thomas Henry Huxley, ein berühmter Biologe, äusserte einmal: «The great tragedy of science [und hier of pseudoscience] - is the slaying of a beautiful hypothesis by an ugly fact.»

\section{Neuraltherapie}

Wieder Edzard Ernst [5]: «Injektionen mit Lokalanästhetika zur Identifikation von Gesundheitsproblemen, Behandlung von Krankheiten, Symptomlinderung ... Novocaininjektionen in Störfelder (biologisch undefiniert) ... dramatische Wirkungen in anderen Körperteilen ... die nichts zu tun haben mit pharmakologischer Wirkung, sondern mit dem autonomen Nervensystem ... sog. Sekundärphänomen.» Injektionen von Lokalanästhetika in ein Schmerzgebiet haben einen voraussagbaren Effekt, hat aber nichts zu tun mit Neuraltherapie.

Die wenigen Studien haben keine überzeugenden Resultate ergeben. Die Neuraltherapie ist biologisch unplausibel, es gibt keine Evidenz.

\section{Häufige Argumente alternativ/ komplementär arbeitender Ärzte}

\section{AK-Ärzte zitieren sinngemäss Shakespeare, wo Hamlet zu Horatio sagt: «There are more things in heaven and earth, Horatio, than are dreamt of in your philosophy." \\ Antwort der normalen Medizin: Wenn sich plausible Ideen in heaven and earth träumen lassen, sind wir}

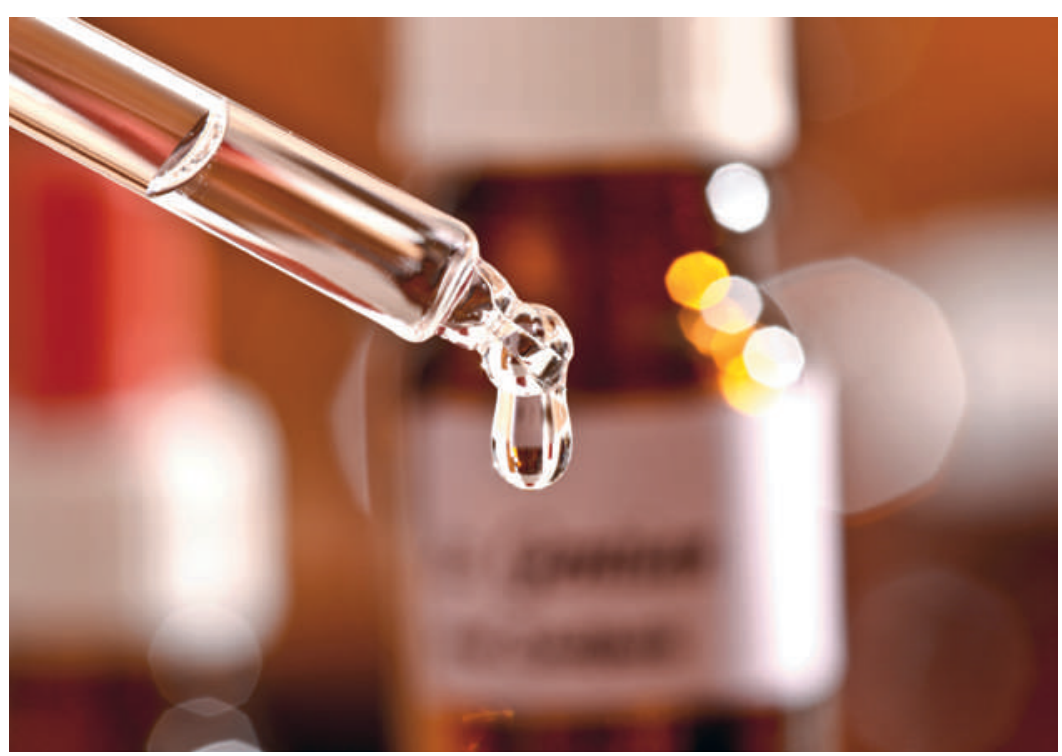

Kann eine Substanz wirken, wenn die letztendlich verwendete Lösung so stark verdünnt ist, dass kein einziges Molekül der Substanz mehr nachweisbar ist? noch so bereit, sie mit wissenschaftlich korrekten Studien zu prüfen. Oder wie der berühmte Physiker Richard Feynman einst sagte: «No matter how beautiful your theory, no matter how clever you are or what your name is, if it disagrees with experiment, then we have no way of telling whether it's right or wrong, and it simply doesn't matter either way.»

\section{Die Wirkung der AK-Medizin kann nur am Indivi- duum geprüft werden.}

Antwort: Bei identischen Endpunkten (s. Einleitung) dürfen ruhig randomisierte Doppelblindstudien an grösseren Patientenzahlen durchgeführt werden. Auch Studien an nur einem Patienten (sog. N-gleich-1-Studien) sind möglich, indem der Patient doppelblind und randomisiert untersucht wird und Phasen mit Verum und Plazebo randomisiert eingesetzt werden.

\section{In der normalen Medizin kommt es zu Selektions- und Interpretations-Vorurteilen und Publikation von positiven Studien mit Zurückhalten von negativen Resultaten durch die Pharmaindustrie.} Antwort: Diese Vorwürfe treffen zu, werden aber streng untersucht, wenn sie ruchbar werden.

\section{AK-Anwendungen sind ganzheitlich und die der Normalmedizin reduktionistisch.}

Antwort: Dieses Argument trifft nicht zu. Erstens wird beispielsweise in der Homöopathie die komplexe Natur des Menschen auf einen einzigen Stoff, der wirken soll, reduziert und "ganzheitlich» nicht definiert. Modelle, in denen das Problem "ganzheitlich" in der Normalmedizin gefunden werden kann, kommen bei Engel [6] und Uexküll [7] vor und in einem Artikel, den der Autor [8] über dieses Problem verfasst hat, nicht aber in AKPublikationen.

\section{AK-Therapien schaden nicht.}

Antwort: Es ist mindestens ein Fall von einem Kind mit Asthma bekannt, bei dem eine normalmedizinische Behandlung durch eine homöopathische ersetzt wurde und das Kind im Status asthmaticus starb. Oder Reisende in malariaverseuchte Länder werden mit einem homöopathischen Medikament zur Prophylaxe versorgt anstatt mit einem als wirksam bekannten Antimalariamittel.

\section{AK-Anwendungen sind auch beim Tier wirksam, es kenne keine Plazebowirkung.}

Antwort: Tiere können auf verschiedene Weise mit Medikamenten konditioniert werden, beispielsweise Pawlows Hunde, die auf den mit der Fütterung assoziierten Klang einer Glocke Magensaft absondern, oder 
Robert Aders Ratten, deren Immunsystem mit Cyclophosphamid intraperitoneal und Sacharin oral konditionierbar ist [9], so dass nach der Konditionierung Saccharin per os allein zur Immunantwortabschwächung führt. Gestützt auf sorgfältige Analysen der Tierhomöopathie ist auch bei diesen keine Wirkung der Homöopathie nachzuweisen (s. Ref. [1], S. 160).

Die Neigung, moderne Erkenntnisse der Physik zur Unterstützung biologisch unplausibler Theorien heranzuziehen, wird bei AK-Ärzten häufig beobachtet. Dabei ist offensichtlich, dass sie zum Beispiel von der zur Rechtfertigung ihrer Theorie herangezogenen Quantenphysik nicht genügend verstehen.

AK-Medizin dient der Verbilligung der Medizinkosten. Antwort: Diese Vermutung ist unbelegt. Da häufig vom Patienten sowohl AK-Ärzte als auch Ärzte der normalen Medizin besucht werden, könnte die Addition sogar zur Verteuerung führen.

\section{Anwendungen sind schon seit Jahrzehnten im Gebrauch und weitverbreitet und deshalb sicher nützlich.}

Antwort: Aderlässe, Quecksilber enthaltende Präparate und Darmspülungen waren jahrzehntelang weitverbreitet und in ihrer Zeit von vielen Ärzten zum Schaden vieler Patienten verwendet worden.

\section{Korrespondenz:}

Rolf Adler

Leiserenweg 4

3122 Kehrsatz

michele.rolf.adler[at]

gmail.com

\section{Fazit}

Es gibt nur eine Medizin. Die Naturwissenschaft vom Menschen ist die Wissenschaft von der Natur des Menschen, und diese ist eine biopsychosoziale. Ihre Erfor- schung kann nur mehr oder weniger ganzheitlich sein, jede Forschung muss sich Beschränkungen in ihrer Planung auferlegen. Erweisen sich Therapien in doppelblinden und randomisierten Studien als über die Plazebowirkung hinaus signifikant wirksam, gehören sie in die (biopsychosoziale) Normalmedizin, sonst in den Papierkorb.

Das Kriterium der Wirksamkeit darf nicht aufgeweicht werden. Ein Lehrstuhl für AK-Medizin ist nur dann gerechtfertigt, wenn der Stelleninhaber nicht einfach die AK-Medizin propagieren will, sondern ihre Ergebnisse zu evaluieren sucht, so wie es Ernst [1] getan hat.

Die ethische Frage, ob die Plazebowirkung biologisch unplausibler Präparate und andere Formen von Therapien für Gesundheitsprobleme herangezogen werden dürfen, muss der Einzelne für sich entscheiden. Für mich beruht die Plazebowirkung auf der Arzt-PatientBeziehung. Ist der Arzt in der Beziehung geschult und erfahren, ist die Verwendung von Plazebo nicht nötig.

\section{Bildnachweis}

(c) MEV Verlag GmbH, Germany

Literatur

1 Singh S, Ernst E. Trick or Treatment. Alternative Medicine on Trial. Corgi Books. 2009;172-6.

2 Adler RH. Die Homöopathie und ihre Beziehung zum Unbewussten des Arztes. Schweiz Med Wschr. 1992;(122):215-6.

3 Frey H et al. Eur J Pediat. 2005;758-67.

4 Adler RH, Herschkowitz N, Minder CE. Eur J Pediat. 2006;(166):509.

5 siehe 1: p. 380.

6 Adler RH, Uexküll Th v, Herrmann JM. The two faces of medical evidence. Swiss Med Weekly. 2002;132:397-400.

7 von Uexküll T. Psychosomatische Medizin; Adler RH et al. (Hg). 7. Aufl., 1. Kapitel: von Uexküll T. München: Urban und Fischer, 2010.

8 Adler RH. Ganzheitlich - der Begriff, sein Missbrauch und einige Klärungen. Schweiz Med Wschr. 1998;128:1075-85.

9 Ader R, Cohen N. Behaviorally conditioned immunosuppression. Psychosom Med. 1975;(37):33. 